\title{
From Bipedal Walking to Quadrupedal Locomotion: Full-Body Dynamics Decomposition for Rapid Gait Generation
}

\author{
Wen-Loong $\mathrm{Ma}^{1}$ and Aaron D. Ames ${ }^{2}$
}

\begin{abstract}
This paper systematically decomposes quadrupeds into bipeds to rapidly generate walking gaits, and then recomposes these gaits to obtain quadrupedal locomotion. We begin by decomposing the full-order, nonlinear and hybrid dynamics of a three-dimensional quadrupedal robot, including its continuous and discrete dynamics, into two bipedal systems that are subject to external forces. Using the hybrid zero dynamics (HZD) framework, gaits for these bipedal robots can be rapidly generated (on the order of seconds) along with corresponding controllers. The decomposition is performed in such a way that the bipedal walking gaits and controllers can be composed to yield dynamic walking gaits for the original quadrupedal robot - the result, therefore, is the rapid generation of dynamic quadruped gaits utilizing the full-order dynamics. This methodology is demonstrated through the rapid generation (3.96 seconds on average) of four stepping-in-place gaits and one ambling gait at $0.35 \mathrm{~m} / \mathrm{s}$ on a quadrupedal robot - the Vision 60, with 36 state variables and 12 control inputs - both in simulation and through outdoor experiments. This suggested a new approach for fast quadrupedal trajectory planning using full-body dynamics, without the need for empirical model simplification, wherein methods from dynamic bipedal walking can be directly applied to quadrupeds.
\end{abstract}

\section{INTRODUCTION}

The control of quadrupedal robots has seen great experimental success in achieving locomotion that is robust and agile, dating back to the seminar work of Rabiert [26]. These results have been achieved despite the fact that quadrupedal robots have more legs, degrees of freedom, and richer context scenarios when compared to their bipedal counterparts. Bipedal robots (while seeing recent successes) still have yet to experimentally demonstrate the dynamic walking behaviors in real-world settings that quadrupeds are now displaying on multiple platforms. Yet, due to the lower degrees of freedom and, importantly, simpler contact interactions with the world, gait generation for bipedal robots based upon the full-order dynamics has a level of rigour not yet present in the quadrupedal locomotion literature (which largely leverages heuristic and reduced-order models). It is this gap between bipedal and quadrupedal robots that this paper attempts to address: can the formal full-order gait generation methods for bipeds be translated to quadrupeds while preserving the positive aspects quadrupedal locomotion?

To achieve walking on quadrupeds, model reduction techniques are widely used for controller design. For example,

*The work of WL. Ma and A. D. Ames are supported by NSF grants 1724464, 1544332 and 1724457.

${ }^{1} \mathrm{WL}$. Ma is with the department of mechanical engineering, California Institute of Technology, Pasadena, CA, 91125. wmalcaltech.edu

${ }^{2} \mathrm{~A}$. Ames is with the faculty of the department of Control + Dynamical Systems, California Institute of Technology, Pasadena, CA, 91125. ames@caltech.edu

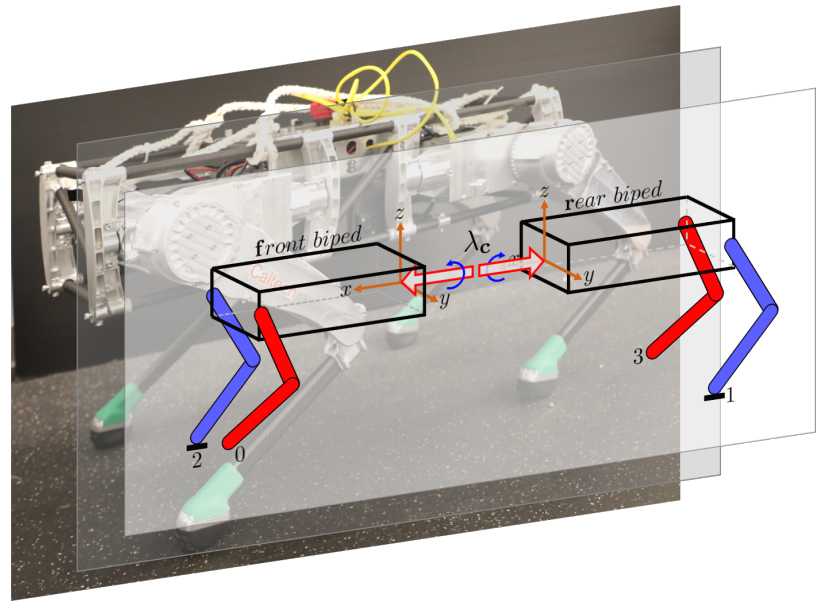

Fig. 1. A conceptual illustration of the full body dynamics decomposition, where the $3 \mathrm{D}$ quadruped - the Vision $60-$ is decomposed into two constrained 3D bipedal robots.

the massless legs [6], [9], linear inverted pendulum model [21], [10] and assuming the 3D quadrupedal motion can be simplified to a planar motion [7], [11] are often utilized methods to mitigate the computational complexity of the quadrupedal dynamics so that online control techniques such as QP, MPC, LQR can be applied [8]. While these methods are very effective in practice, it often requires some add-on layers of parameter tuning due to the gap between model and reality. This is particularly prevalent for bigger and heavier robots whose physical properties play a larger role.

In the context of bipedal robots, due to their inherently unstable nature, detailed model and rigorous controller design have been long been developed. A specific methodology that leverages the full-order dynamics of the robot to make formal guarantees is Hybrid Zero Dynamics (HZD) [31], [5], [2] which has seen success experimentally for both walking and running [29], [23], [27]. A key to this success has been the recent developments in rapid HZD gait generation using collocation methods [18], with the ability to generate gaits for high-dimensional robots in some cases in seconds [20]. Recently, the HZD framework was translated to quadrupedal robots both for gait generation and controller design [22], [3]. Although the end result was the ability generate walking, ambling and trotting for the full-order model, the high dimensional and complex contacts of the system made the gait generation complex with the fast gait being generated in 43 seconds and hours of post-processing needed to guarantee stability. The goal of this paper is, therefore, to translate the positive aspects of HZD gait generation to quadrupeds while 


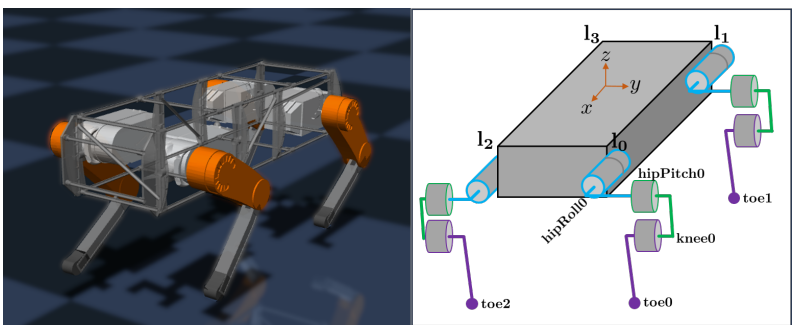

Fig. 2. On the left is the the robot in MuJoCo, and on the right is the illustration of the configuration coordinates for the robot. The leg indices $l_{*}$ are shown on the vertices of the body link. Each leg has three actuated joints and equipped with a point contact toe.

mitigating the aforementioned drawbacks.

Pioneers in robotics have observed the correlation between bipedal and quadrupedal locomotion, for example, [26], [25] applied a variety bipedal gaits on quadrupedal robots and [11], [12] provided stability analysis for a planar abstract hopping robot. However, these results still rely on the model reduction methods such as the $2 \mathrm{D}$ modelling and massless legs. Additionally, the focus were on composing bipedal controllers to stabilize quadrupedal locomotion rather than decomposing the dynamics of quadrupeds to bipedal systems while considering the evolution of the internal connection wrench. Notably, they lack a systematic approach of producing trajectories for the control of bipeds as a decomposed system from the quadrupeds.

The main contribution of this paper is the exact decomposition of quadrupeds into bipeds, wherein gaits can be rapidly generated and composed to be realized on the quadruped from which they were derived. Specifically, the main results of this paper are twofold: 1) A systematic decomposition of the three-dimensional full body dynamics of a quadruped, which involved both the continuous and discrete dynamics, into two bipedal hybrid systems subject to external forces; 2) An optimization algorithm that generates gaits for the bipedal system rapidly utilize the framework of HZD, wherein they can then be composed to yield gaits on the quadruped. The end result is that we are able to generate various bipedal gaits that can be recomposed to quadrupedal behaviors within seconds, and these behaviors are implemented successfully in simulation and experimentally in outdoor environments.

This paper is organized as follows: Section II introduces the general idea of decomposing the hybrid full body dynamics of a quadrupedal robot into lower dimensional half body dynamics of two identical bipeds. Based on this, we produced trajectories for stepping-in-place and ambling on a quadrupedal robot Vision60 in Section III. The efficiency is shown by an analysis of its computation performance compared against the full-body dynamics optimization for gait generation. In Section IV, we validate the resultant trajectories in $\mathrm{MuJoCo}[30]$ (a commercial simulation environment), and five outdoor experiments to demonstrate the feasibility of theses trajectories that are built based on decomposed bipedal dynamics. Section V concludes the paper and proposes several future directions.

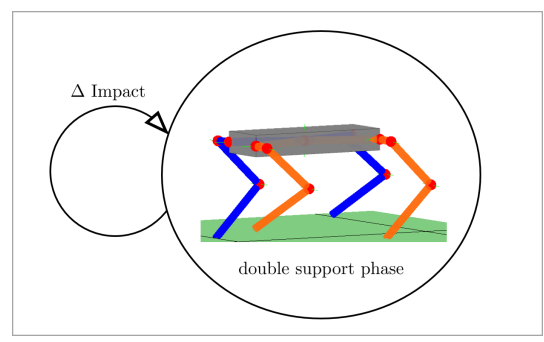

Fig. 3. The cyclic directed graph for the single-domain hybrid dynamics of the diagonally supporting ambling behavior.

\section{DYNAMICS DECOMPOSITION}

In this section, we decompose the full body dynamics and control of quadrupedal robots into two identical bipedal systems. The nonlinear model of quadrupedal locomotion is a hybrid dynamical system, which is an alternating sequence of continuous- and discrete-time dynamics. The order of the sequence is dictated by contact events.

\section{A. Full body dynamics of a quadruped}

This full body dynamics of quadrupedal robots have been detailed in [22] and will be briefly revisited here to setup the problem properly. Note that in this section, we only focus on the most popular quadrupedal robotic behavior the diagonally supporting amble (see Fig. 3). We refer the readers to [22] for other contact scenarios and the multidomain setups.

1) State space and inputs: The robot begin considered the Vision 60 V3.2 in Fig. 2 - is composed of 13 links: a body link and 4 legs, each of which has three sublinks the hip, upper and lower links. Utilizing the floating base convention [15], the configuration space is chosen as $q=$ $\left(q_{b}^{T}, \theta_{0}^{T}, \theta_{1}^{T}, \theta_{2}^{T}, \theta_{3}^{T}\right)^{T} \in \mathcal{Q} \subset \mathbb{R}^{18}$, where $q_{b} \in \mathbb{R}^{3} \times \mathrm{SO}(3)$ represents the Cartesian position and orientation of the body linkage, and $\theta_{i} \in \mathbb{R}^{3}$ represents the three joints: hip roll, hip pitch and knee on the leg $i \in\{0,1,2,3\}$. All of these leg joints are actuated, with torque inputs $u_{i} \in \mathbb{R}^{3}$. This yields the system's total DOF $n=18$ and control inputs $u=\left(u_{0}^{T}, u_{1}^{T}, u_{2}^{T}, u_{3}^{T}\right)^{T} \in \mathbb{R}^{m}, m=12$. Further, we can define the state space $\mathcal{X}=T \mathcal{Q} \subseteq \mathbb{R}^{2 n}$ with the state vector $x=\left(q^{T}, \dot{q}^{T}\right)^{T}$, where $T \mathcal{Q}$ is the tangent bundle of the configuration space $\mathcal{Q}$.

2) Continuous dynamics: The continuous-time dynamics in Fig. 3. when toe1 and toe 2 are on the ground, are modelled as constrained dynamics:

$$
\left\{\begin{array}{l}
D(q) \ddot{q}+H(q, \dot{q})=B u+J_{1}^{T}(q) \lambda_{1}+J_{2}^{T}(q) \lambda_{2} \\
J_{1}(q) \ddot{q}+\dot{J}_{1}(q, \dot{q}) \dot{q}=0 \\
J_{2}(q) \ddot{q}+\dot{J}_{2}(q, \dot{q}) \dot{q}=0
\end{array}\right.
$$

when $x \in \mathcal{D}$, where

$$
\mathcal{D}:=\left\{x \in \mathcal{X}: \dot{h}_{1}(q, \dot{q})=\dot{h}_{2}(q, \dot{q})=0, h_{z_{1}}(q)=h_{z_{2}}(q)=0\right\} .
$$

In this formulation, we utilize the following notation: $D(q) \in$ $\mathbb{R}^{n \times n}$ is the inertia-mass matrix; $H(q, \dot{q}) \in \mathbb{R}^{n}$ contains Coriolis forces and gravity terms; $h_{1}(q), h_{2}(q) \in \mathbb{R}^{3}$ are 
the Cartesian positions of toe 1 and toe 2 and their Jacobians are $J_{*}=\partial h_{*} / \partial q ; h_{z_{1}}(q), h_{z_{2}}(q)$ are these toes' height; $\lambda_{1}, \lambda_{2}$ are the ground reaction force on toe1 and toe2; $B \in \mathbb{R}^{n \times m}$ is the actuation matrix. Essentially, we use a set of differential algebra equations (DAEs) to describe the dynamics of the quadrupedal robot that is subject to two holonomic constraints on toe1 and toe2.

3) The discrete impact dynamics: On the boundary of domain $\mathcal{D}$ we impose discrete-time dynamics to encode the impact dynamics as toe 0 and toe 3 impact the ground (and suppressing the dependence of $D$ and $J_{*}$ on $q$ and $\dot{q}$ ):

$$
\left\{\begin{array}{l}
D\left(\dot{q}^{+}-\dot{q}^{-}\right)=J_{0}^{T} \Lambda_{0}+J_{3}^{T} \Lambda_{3} \\
J_{0} \dot{q}^{+}=0 \\
J_{3} \dot{q}^{+}=0
\end{array}\right.
$$

by using conservation of momentum while satisfying the next domain's holonomic constraints, which is that toe 0 and toe 3 stay on the ground after the impact event. We denoted $\dot{q}^{-}$and $\dot{q}^{+}$as the pre- and pose-impact velocity terms, $\Lambda_{0}, \Lambda_{3} \in \mathbb{R}^{3}$ are the impulses exerted on toe 0 and toe 3 .

\section{B. Continuous dynamics decomposition}

We now decompose the quadrupedal full body dynamics into two bipedal robots. First, as shown in Fig. 1, the openloop dynamics can be equivalently written as (and suppressing the dependence on $q$ and $\dot{q}$ for notational simplicity):

$$
\text { OL-Dyn: }\left\{\begin{array}{l}
D_{\mathrm{f}} \ddot{q}_{\mathrm{f}}+H_{\mathrm{f}}=J_{\mathrm{f}_{2}}^{T} \lambda_{2}+B_{\mathrm{f}} u_{\mathrm{f}}-J_{c}^{T} \lambda_{c} \\
J_{\mathrm{f}_{2}} \ddot{q}_{\mathrm{f}}+\dot{J}_{\mathrm{f}_{2}} \dot{q}_{\mathrm{f}}=0 \\
D_{\mathrm{r}} \ddot{q}_{\mathrm{r}}+H_{\mathrm{r}}=J_{\mathrm{r}_{1}}^{T} \lambda_{1}+B_{\mathrm{r}} u_{\mathrm{r}}+J_{c}^{T} \lambda_{c} \\
J_{\mathrm{r}_{1}} \ddot{q}_{\mathrm{r}}+\dot{J}_{\mathrm{r}_{1}} \dot{q}_{\mathrm{r}}=0 \\
\ddot{q}_{b_{\mathrm{r}}}-\ddot{q}_{b_{\mathrm{f}}}=0
\end{array}\right.
$$

wherein we utilized the following notation: $q_{b_{\mathrm{r}}}, q_{b_{\mathrm{f}}} \in \mathbb{R}^{3} \times$ $S O(3)$ are the coordinates for the body linkages of the front and rear bipeds (see Fig. 11; $q_{\mathrm{f}}=\left(q_{b_{\mathrm{f}}}^{T}, \theta_{0}^{T}, \theta_{2}^{T}\right)^{T}$ and $q_{\mathrm{r}}=\left(q_{b_{\mathrm{r}}}^{T}, \theta_{1}^{T}, \theta_{3}^{T}\right)^{T}$ are the configuration coordinates for the front and rear bipeds; $D_{\mathrm{f}}\left(q_{\mathrm{f}}\right), D_{\mathrm{r}}\left(q_{\mathrm{r}}\right) \in \mathbb{R}^{12 \times 12}$ are the inertia-mass matrices of the front and rear bipedal robots; The Jacobians $J_{\mathrm{f}_{2}}=\partial h_{\mathrm{f}_{2}} / \partial q_{\mathrm{f}}, J_{\mathrm{r}_{1}}=\partial h_{\mathrm{r}_{1}} / \partial q_{\mathrm{r}}$ with the Cartesian positions of toe $1-h_{\mathrm{f}_{2}}\left(q_{\mathrm{f}}\right)$ and toe $2-h_{\mathrm{r}_{1}}\left(q_{\mathrm{r}}\right)$; The Jacobian matrix for the connection constraint (7) is $J_{c}=\partial\left(q_{b_{\mathrm{r}}}-q_{b_{\mathrm{f}}}\right) / \partial q_{\mathrm{f}} ; u_{\mathrm{f}}=\left(u_{0}^{T}, u_{2}^{T}\right)^{T}$ and $u_{\mathrm{r}}=\left(u_{1}^{T}, u_{3}^{T}\right)^{T}$. Note that the fact the Cartesian position of toe 2 only depends on $q_{\mathrm{f}}$ is the result of the floating base coordinate convention.

Proposition 1. The dynamical system (OL-Dyn) is equivalent to the system (1).

Proof. We can write (3) and (5) as:

$$
\begin{aligned}
& {\left[\begin{array}{ccc}
D_{b_{\mathrm{f}}} & D_{b_{0}} & D_{b_{2}} \\
D_{b_{0}}^{T} & D_{0} & 0 \\
D_{b_{2}}^{T} & 0 & D_{2}
\end{array}\right]\left[\begin{array}{c}
\ddot{q}_{b_{\mathrm{f}}} \\
\ddot{q}_{0} \\
\ddot{q}_{2}
\end{array}\right]+\left[\begin{array}{c}
H_{b_{\mathrm{f}}} \\
H_{0} \\
H_{2}
\end{array}\right]=B_{\mathrm{f}} u_{\mathrm{f}}+J_{\mathrm{f}_{2}}^{T} \lambda_{2}-J_{c}^{T} \lambda_{c}} \\
& {\left[\begin{array}{ccc}
D_{b_{\mathrm{r}}} & D_{b_{1}} & D_{b_{3}} \\
D_{b_{1}}^{T} & D_{1} & 0 \\
D_{b_{3}}^{T} & 0 & D_{3}
\end{array}\right]\left[\begin{array}{c}
\ddot{q}_{b_{\mathrm{r}}} \\
\ddot{q}_{1} \\
\ddot{q}_{3}
\end{array}\right]+\left[\begin{array}{c}
H_{b_{\mathrm{r}}} \\
H_{1} \\
H_{3}
\end{array}\right]=B_{\mathrm{r}} u_{\mathrm{r}}+J_{\mathrm{r}_{1}}^{T} \lambda_{1}+J_{c}^{T} \lambda_{c}}
\end{aligned}
$$

Expanding these expressions yields:

$$
\begin{aligned}
& {\left[\begin{array}{ccccc}
D_{b_{\mathrm{f}}} & D_{b_{0}} & 0 & D_{b_{2}} & 0 \\
D_{b_{0}}^{T} & D_{0} & 0 & 0 & 0 \\
0 & 0 & 0 & 0 & 0 \\
D_{b_{2}}^{T} & 0 & 0 & D_{2} & 0 \\
0 & 0 & 0 & 0 & 0
\end{array}\right]\left[\begin{array}{c}
\ddot{q}_{b_{\mathrm{f}}} \\
\ddot{q}_{0} \\
\ddot{q}_{1} \\
\ddot{q}_{2} \\
\ddot{q}_{3}
\end{array}\right]+\left[\begin{array}{c}
H_{b_{\mathrm{f}}} \\
H_{0} \\
0 \\
H_{2} \\
0
\end{array}\right]=\left[\begin{array}{c}
-\lambda_{c} \\
u_{0} \\
0 \\
u_{2} \\
0
\end{array}\right]+J_{2}^{T} \lambda_{2},} \\
& {\left[\begin{array}{ccccc}
D_{b_{\mathrm{r}}} & 0 & D_{b_{1}} & 0 & D_{b_{3}} \\
0 & 0 & 0 & 0 & 0 \\
D_{b_{1}}^{T} & 0 & D_{1} & 0 & 0 \\
0 & 0 & 0 & 0 & 0 \\
D_{b_{3}}^{T} & 0 & 0 & 0 & D_{3}
\end{array}\right]\left[\begin{array}{c}
\ddot{q}_{b_{\mathrm{r}}} \\
\ddot{q}_{0} \\
\ddot{q}_{1} \\
\ddot{q}_{2} \\
\ddot{q}_{3}
\end{array}\right]+\left[\begin{array}{c}
H_{b_{\mathrm{r}}} \\
0 \\
H_{1} \\
0 \\
H_{3}
\end{array}\right]=\left[\begin{array}{c}
\lambda_{c} \\
0 \\
u_{1} \\
0 \\
u_{3}
\end{array}\right]+J_{1}^{T} \lambda_{1} .}
\end{aligned}
$$

Now, these two equations can be combined together with the holonomic connection constraint $q_{b_{\mathrm{f}}}-q_{b_{\mathrm{r}}} \equiv \sqrt{1}$, with the end result being the dynamics given in (1). It is worthwhile to note that the terms that appeared in these equations can be verified using traditional rigid body dynamics and the corresponding details of the structure and necessary properties of the inertia-mass matrices can be found from the branch induced sparsity [13].

Note that (7) can be equivalently replaced by summating the first 6 equations of $(3)$ and $(5)$ :

$$
\left(D_{b_{\mathrm{f}}}+D_{b_{\mathrm{r}}}\right) \ddot{q}_{b_{i}}+\sum_{j=0}^{3} D_{b j} \ddot{q}_{j}+H_{b_{\mathrm{f}}}+H_{b_{\mathrm{r}}}=J_{\mathrm{r}_{1}, b}^{T} \lambda_{1}+J_{\mathrm{f}_{2}, b}^{T} \lambda_{2}
$$

Denoted by: $\quad h_{c}\left(q_{\mathrm{f}}, \dot{q}_{\mathrm{f}}, \ddot{q}_{\mathrm{f}}, \lambda_{2}, q_{\mathrm{r}}, \dot{q}_{\mathrm{r}}, \ddot{q}_{\mathrm{r}}, \lambda_{1}\right)=0$

where $i=\mathrm{f}, \mathrm{r}$ and $J_{\mathrm{r}_{1}, b}, J_{\mathrm{f}_{2}, b}$ are the corresponding submatrices:

$$
J_{\mathrm{r}_{1}}=\left[\begin{array}{ll}
J_{\mathrm{r}_{1}, b} & J_{\mathrm{r}_{1}, \theta}
\end{array}\right], \quad J_{\mathrm{f}_{2}}=\left[\begin{array}{ll}
J_{\mathrm{f}_{2}, b} & J_{\mathrm{f}_{2}, \theta}
\end{array}\right] .
$$

Consider a system obtained from (3), (4), and (8) which defines the front biped (see Fig. 11):

$$
\text { (f) }:\left\{\begin{array}{l}
D_{\mathrm{f}} \ddot{q}_{\mathrm{f}}+H_{\mathrm{f}}=J_{\mathrm{f}_{2}}^{T} \lambda_{2}+B_{\mathrm{f}} u_{\mathrm{f}}-J_{c}^{T} \lambda_{c} \\
J_{\mathrm{f}_{2}} \ddot{q}_{\mathrm{f}}+\dot{J}_{\mathrm{f}_{2}} \dot{q}_{\mathrm{f}}=0 \\
h_{c}\left(q_{\mathrm{f}}, \dot{q}_{\mathrm{f}}, \ddot{q}_{\mathrm{f}}, \lambda_{2}, q_{\mathrm{r}}, \dot{q}_{\mathrm{r}}, \ddot{q}_{\mathrm{r}}, \lambda_{1}\right)=0
\end{array}\right.
$$

which is a dynamical system subject to the feedforward terms $\left(q_{\mathrm{r}}, \dot{q}_{\mathrm{r}}, \ddot{q}_{\mathrm{r}}, \lambda_{1}\right)$. The dynamics of the rear biped, (r), can be similarly obtained using (5), (6), and (8). We have thus decomposed the dynamics of a quadrupedal robot (1) to two bipedal dynamical systems (f) and (r), as illustrated by Fig. 1 .

Example 1. The methodology of dynamics decomposition can be extended to other mechanical systems. We illustrate this idea by showing it on the simple example illustrated in Fig. 4. Note that each subsystem is not subject to any constraints. The half-body dynamics of the single cart with an inverted pendulum are given by:

$$
\left[\begin{array}{cc}
M+m & -m l \cos \theta_{i} \\
-m l \cos \theta_{i} & m l^{2}
\end{array}\right]\left[\begin{array}{c}
\ddot{x}_{i} \\
\ddot{\theta}_{i}
\end{array}\right]+\left[\begin{array}{c}
m l \dot{\theta}_{i} \sin \theta_{i} \\
-m g l \sin \theta_{i}
\end{array}\right]=\left[\begin{array}{c}
\lambda_{c} \\
u_{i}
\end{array}\right]
$$

where $i \in\{\mathrm{f}, \mathrm{r}\}$. We can use a joint-space $P D$ controller $u_{\mathrm{f}}\left(\theta_{\mathrm{f}}, \dot{\theta}_{\mathrm{f}}\right), u_{\mathrm{r}}\left(\theta_{\mathrm{r}}, \dot{\theta}_{\mathrm{r}}\right)$ to achieve a desired behavior such that

\footnotetext{
${ }^{1}$ The operator $\equiv$ is referring to that the terms on the left and right side of the equation equals to each other, both for their 0 and 1 st order derivatives.
} 


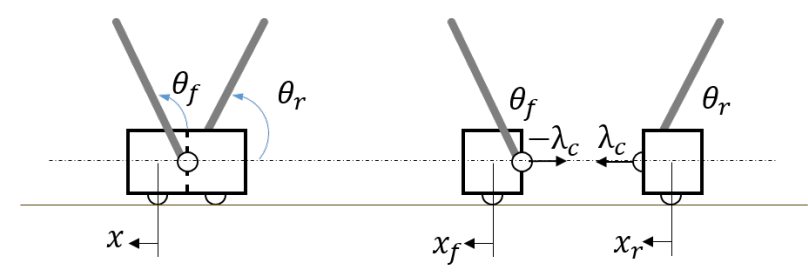

Fig. 4. The full body dynamics are composed of two invert pendulum carts, both rotational joints are actuated with inputs $u_{\mathrm{f}}, u_{\mathrm{r}}$. The mass of the cart is $2 M$ and each of the pendulum weights $m$ with length $l$.

the two invert pendulums vibrate symmetrically, i.e., $\theta_{\mathrm{f}}=$ $-\theta_{\mathrm{r}}+\pi$. Then from (8) we can have

$$
(2 M+2 m) \ddot{x}_{i}=0
$$

which yields $\ddot{x}_{i}=0$ and the internal connection force: $\lambda_{c}=-m l \cos \theta_{1} \ddot{\theta}_{1}+m l \dot{\theta}_{1} \sin \theta_{1}$. This means that when the two invert pendulum are moving symmetrically, the cart will have zero acceleration. This physics example is rather trivial, but it suggested some insights on why a bipedal system (or a single invert pendulum) is difficult to stabilize while a quadrupedal (or a parallel double invert pendulum) is easier to remain stationary even though it has more DOF.

\section{Control decomposition}

To achieve the desired behavior - diagonally symmetric amble — we now add a controller to track desired timebased trajectories. The algorithm we used to produce these trajectories will be detailed in the next section. We define outputs for the biped $i$ with $i \in\{\mathrm{f}, \mathrm{r}\}$ as $y_{i}=y_{i}^{a}\left(q_{i}\right)-\mathcal{B}_{i}(t)$, with $t$ the time and $\mathcal{B}(t)$ a 5 th order Beźier polynomial. For a simple case study, we chose $y_{i}^{a}\left(q_{i}\right)$ as the actuated joints:

$$
y_{\mathrm{f}}^{a}=\left[\begin{array}{l}
\theta_{0} \\
\theta_{2}
\end{array}\right], \quad y_{\mathrm{r}}^{a}=\left[\begin{array}{l}
\theta_{1} \\
\theta_{3}
\end{array}\right] .
$$

By imposing that the output dynamics on $y_{i}$ act like those of a linear system (as can be enforced through control), we have the closed-loop dynamics of the decomposed bipeds subject to control as follows:

$$
\begin{aligned}
& \left\{\begin{array}{l}
D_{\mathrm{f}} \ddot{q}_{\mathrm{f}}+H_{\mathrm{f}}=J_{\mathrm{f}_{2}}^{T} \lambda_{2}+B_{\mathrm{f}} u_{\mathrm{f}}^{c}-J_{c}^{T} \lambda_{c} \\
J_{\mathrm{f}_{2}} \ddot{q}_{\mathrm{f}}+\dot{J}_{\mathrm{f}_{2}} \dot{q}_{\mathrm{f}}=0 \\
\ddot{y}_{\mathrm{f}}=k_{1} \dot{y}_{\mathrm{f}}+k_{2} y_{\mathrm{f}} \\
h_{c}\left(q_{\mathrm{f}}, \dot{q}_{\mathrm{f}}, \ddot{q}_{\mathrm{f}}, \lambda_{2}, q_{\mathrm{r}}, \dot{q}_{\mathrm{r}}, \ddot{q}_{\mathrm{r}}, \lambda_{1}\right)=0
\end{array}\right. \\
& \left\{\begin{array}{l}
D_{\mathrm{r}} \ddot{q}_{\mathrm{r}}+H_{\mathrm{r}}=J_{\mathrm{r}_{1}}^{T} \lambda_{1}+B_{\mathrm{r}} u_{\mathrm{r}}^{c}+J_{c}^{T} \lambda_{c} \\
J_{\mathrm{r}_{1}} \ddot{q}_{\mathrm{r}}+\dot{J}_{\mathrm{r}_{1}} \dot{q}_{\mathrm{r}}=0 \\
\ddot{y}_{\mathrm{r}}=k_{1} \dot{y}_{\mathrm{r}}+k_{2} y_{\mathrm{r}} \\
h_{c}\left(q_{\mathrm{f}}, \dot{q}_{\mathrm{f}}, \ddot{q}_{\mathrm{f}}, \lambda_{2}, q_{\mathrm{r}}, \dot{q}_{\mathrm{r}}, \ddot{q}_{\mathrm{r}}, \lambda_{1}\right)=0
\end{array}\right.
\end{aligned}
$$

where $u_{i}^{c}$ are the control inputs imposing the output dynamics as desired. The output dynamics implemented here is an implicit version of input-output feedback linearization, details of this implementation can be found in [16], [4].

However, to design a proper trajectory and determine the control inputs for the biped (f), we need to know all of the feedforward terms $\left(q_{\mathrm{r}}, \dot{q}_{\mathrm{r}}, \ddot{q}_{\mathrm{r}}, \lambda_{1}\right)$ for the time $t \in[0, T]$, with $T$ the time duration of a step. Therefore we encode a correlation between the desired trajectories of the front and rear bipeds as follows:

$$
\mathcal{B}_{\mathrm{r}}(t)=M \mathcal{B}_{\mathrm{f}}(t)+b
$$

which imposes a diagonally symmetric gait characteristic via a constant matrix $M$. Specifically, this means that leg3's movement is a mirror of leg0 and leg1's movement is a mirror of leg2. On the zero dynamics (ZD) surface [28] where $y_{i}\left(q_{i}\right) \equiv 0$, together with the connection constraint $q_{b_{\mathrm{f}}} \equiv q_{b_{\mathrm{r}}}$, we have the following correlation on the ZD surface:

$$
q_{\mathrm{r}} \equiv A q_{\mathrm{f}}+b, \quad \text { where } A=\left[\begin{array}{ll}
I & \\
& M
\end{array}\right] \text {. }
$$

In addition, to determine $\lambda_{1}$ of the biped (r), we also need to impose the constraint (6), wherein system (10) becomes:

$$
\left\{\begin{array}{l}
D_{\mathrm{f}} \ddot{q}_{\mathrm{f}}+H_{\mathrm{f}}=J_{\mathrm{f}_{2}}^{T} \lambda_{2}+B_{\mathrm{f}} u_{\mathrm{f}}^{c}-J_{c}^{T} \lambda_{c} \\
J_{\mathrm{f}_{2}} \ddot{q}_{\mathrm{f}}+\dot{J}_{\mathrm{f}_{2}} \dot{q}_{\mathrm{f}}=0 \\
\ddot{y}_{\mathrm{f}}=k_{1} \dot{y}_{\mathrm{f}}+k_{2} y_{\mathrm{f}}=0 \\
J_{\mathrm{r}_{1}} A \ddot{q}_{\mathrm{f}}+\dot{J}_{\mathrm{r}_{1}} A \dot{q}_{\mathrm{f}}=0 \\
h_{c}\left(q_{\mathrm{f}}, \dot{q}_{\mathrm{f}}, \ddot{q}_{\mathrm{f}}, \lambda_{2}, A q_{\mathrm{f}}+b, A \dot{q}_{\mathrm{r}}, A \ddot{q}_{\mathrm{r}}, \lambda_{1}\right)=0
\end{array}\right.
$$

We can subtract the dynamics of (f) from [8] to further simplify the expression to

$$
\text { CL-Dyn-f: }\left\{\begin{array}{l}
D_{\mathrm{f}} \ddot{q}_{\mathrm{f}}+H_{\mathrm{f}}=J_{\mathrm{f}_{2}}^{T} \lambda_{2}+B_{\mathrm{f}} u_{\mathrm{f}}^{c}-J_{c}^{T} \lambda_{c} \\
\ddot{y}_{\mathrm{f}}=0 \\
J_{\mathrm{f}_{2}} \ddot{q}_{\mathrm{f}}+\dot{J}_{\mathrm{f}_{2}} \dot{q}_{\mathrm{f}}=0 \\
J_{\mathrm{r}_{1}} A \ddot{q}_{\mathrm{f}}+\dot{J}_{\mathrm{r}_{1}} A \dot{q}_{\mathrm{f}}=0 \\
\hat{D}_{\mathrm{f}} A \ddot{q}_{\mathrm{f}}+\hat{H}_{\mathrm{r}}=J_{\mathrm{r}_{1}, b}^{T} \lambda_{1}
\end{array}\right.
$$

with $\hat{D}_{\mathrm{f}} \in \mathbb{R}^{6 \times 12}, \hat{H}_{\mathrm{f}} \in \mathbb{R}^{6}$ the first 6 rows of $D_{\mathrm{f}}$ and $H_{\mathrm{f}}$, respectively. We now have the decomposed dynamics of system (f) that is independent from the feedforward terms. We can view this system as a dynamical system (14) subject to one virtual constraint (15) with inputs $u_{\mathrm{f}}^{c}$ and three mechanical constraints (16), (17), and (18) with inputs $\lambda_{1}$, $\lambda_{2}$, and $\lambda_{c}$.

\section{Impact dynamics of the decomposed system}

With the continuous dynamics written as (OL-Dyn), we can similarly expand the impact dynamics (2) as:

$$
\left\{\begin{array}{l}
D_{\mathrm{f}}\left(\dot{q}_{\mathrm{f}}^{+}-\dot{q}_{\mathrm{f}}^{-}\right)=J_{\mathrm{f}_{2}}^{T} \Lambda_{2}-J_{c}^{T} \Lambda_{c} \\
J_{\mathrm{f}_{2}} \dot{q}_{\mathrm{f}}^{+}=0 \\
D_{\mathrm{r}}\left(\dot{q}_{\mathrm{r}}^{+}-\dot{q}_{\mathrm{r}}^{-}\right)=J_{\mathrm{r}_{1}}^{T} \Lambda_{1}+J_{c}^{T} \Lambda_{c} \\
J_{\mathrm{r}_{1}} \dot{q}_{\mathrm{r}}^{+}=0 \\
J_{c}\left(\dot{q}_{\mathrm{r}}^{+}-\dot{q}_{\mathrm{f}}^{+}\right)=0
\end{array}\right.
$$

The proof is similar to that of continuous dynamics decomposition and thus omitted. On the ZD surface, where both of the bipedal systems (f) and (r) have zero tracking errors (we will use an optimization algorithm to determine those gaits that are hybrid invariant, i.e., have hybrid zero dynamics (HZD) [14], [24], [4]), we then have:

$$
q_{\mathrm{r}}^{-}=A q_{\mathrm{f}}^{-}+b, \quad q_{\mathrm{r}}^{+}=A q_{\mathrm{f}}^{+}+b
$$



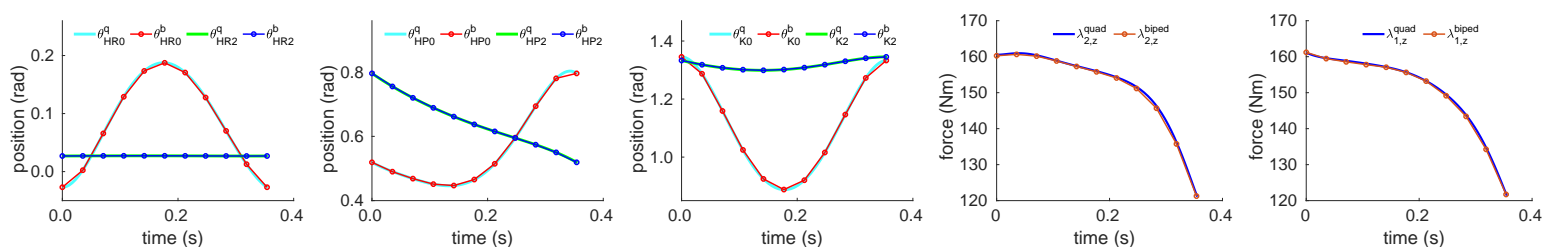

Fig. 5. A comparison between the solution of bipedal walking dynamics obtained from the decomposition based optimization and a simulated step of the full-order quadrupedal dynamics using the composed bipedal gaits; here MATLAB ODE 45 was used.

The impact dynamics of the decomposed system can therefore be obtained:

$$
\begin{gathered}
\Delta:\left\{\begin{array}{l}
D_{\mathrm{f}}\left(\dot{q}_{\mathrm{f}}^{+}-\dot{q}_{\mathrm{f}}^{-}\right)=J_{\mathrm{f}_{2}}^{T} \Lambda_{2}-J_{c}^{T} \Lambda_{c} \\
J_{\mathrm{f}_{2}} \dot{q}_{\mathrm{f}}^{+}=0 \\
D_{\mathrm{r}} A\left(\dot{q}_{\mathrm{f}}^{+}-\dot{q}_{\mathrm{f}}^{-}\right)=J_{\mathrm{r}_{1}}^{T} \Lambda_{1}+J_{c}^{T} \Lambda_{c} \\
J_{\mathrm{r}_{1}} A \dot{q}_{\mathrm{f}}^{+}=0
\end{array}\right. \\
\Leftrightarrow\left[\begin{array}{cccc}
D_{\mathrm{f}} & -J_{\mathrm{f}_{2}}^{T} & 0 & J_{c}^{T} \\
J_{\mathrm{f}_{2}} & 0 & 0 & 0 \\
D_{\mathrm{r}} A & 0 & -J_{\mathrm{r}_{1}}^{T} & -J_{c}^{T} \\
J_{\mathrm{r}_{1}} A & 0 & 0 & 0
\end{array}\right]\left[\begin{array}{c}
\dot{q}_{\mathrm{f}}^{+} \\
\Lambda_{1} \\
\Lambda_{2} \\
\Lambda_{c}
\end{array}\right]=\left[\begin{array}{c}
D_{\mathrm{f}} \dot{q}_{\mathrm{f}}^{-} \\
0 \\
D_{\mathrm{r}} A \dot{q}_{\mathrm{f}}^{-} \\
0
\end{array}\right]
\end{gathered}
$$

One may notice that the system (24) has $12+3+12+3=$ 30 equations but only $12+3+3+6=24$ unknowns. Theoretically we can removed 6 equations from this overdetermined system to offload some computational cost. However this is not desirable in practice, because this manipulation can result in numerically ill-posed computation such that it loses numerical stability. This can be more severe for robots with lighter legs. Moreover, the implicit optimization method discussed in the next section can solve this system accurately and efficiently without this manipulation.

\section{Decomposition BASED TRAJECTORY OPTIMIZATION}

Past work has investigated the formal analysis and controller design for the full body dynamics of quadrupeds [3], [22]. Although we were able to produce trajectories that are stable solutions to the closed-loop multi-domain dynamics for walking, ambling and trotting, the computational complexity make realizing these methods difficult in practice: it typically takes minutes to generate a trajectory and hours to post-process the stability properties. However, by using the dynamics decomposition method, we are able to produce bipedal walking gaits that can be composed to obtain quadrupedal locomotion while maintaining the efficiency of computing the lower dimensional dynamics of bipedal robots. In this section, we will detail this process using a nonlinear programming (NLP).

Given the constrained bipedal dynamics (CL-Dyn-f) and the impact dynamics 24, the target is to find a solution to the closed-loop dynamical system as shown in Fig. 3 efficiently. The nonlinear programming is formulated as:

$$
\begin{aligned}
\min _{\mathbf{Z}} & \sum_{i=1}^{2 N+1}\left\|\dot{q}_{b_{\mathrm{f}}}\right\|_{2}^{2} \\
\text { s.t. } & \text { C1. dynamics (CL-Dyn-f) } \\
& \text { C2. collocation constraints } \\
& \text { C3. impact dynamics 20, 22 } \\
& \text { C4. periodic continuity } \\
& \text { C5. physical feasibility }
\end{aligned}
$$

$$
\begin{array}{r}
i=1,3, \ldots 2 N+1 \\
i=2,4, \ldots 2 N \\
i=2 N+1 \\
i=1,2 N+1 \\
i=1,2, \ldots 2 N+1
\end{array}
$$

with the following notation: $2 N+1=13$ is the total number of collocation grids; the decision variable $\mathbf{Z}=\left(\alpha, x_{i}, \dot{x}_{i}, u_{i}\right)$; and $\alpha \in \mathbb{R}^{36}$ are the static parameters for the Beźier polynomial that defines the desired trajectory $\mathcal{B}_{\mathrm{f}}(t) ; x_{i}, \dot{x}_{i}, u_{i}$ are the corresponding quantities at time $t_{i}$ with $t_{2 N+1}=T$.

In short, the cost function is to minimize the body linkage's vibration rate since a more static torso movement is desirable for experiments. The constraints C1-C3 solve the hybrid dynamics of bipedal robots subject to external forces. More details can be found in [17]. The constraint C4 enforced state continuity through a edge: $\dot{q}_{1}=\Delta \dot{q}_{2 N+1}$ from (24). C5 imposed some feasibility conditions on the dynamics, including torque limits $\left\|u_{i}\right\|_{\infty} \leq 50$, joint reachable space limitation $\left(q_{i}, \dot{q}_{i}\right) \in \mathcal{X}$, foot clearance and the friction pyramid conditions.

Once the optimization (25) converged to a set of parameters $\alpha$ for the front bipedal robots' walking gait $\mathcal{B}_{\mathrm{f}}(t)$, we can use (12) to obtain the trajectory for the rear biped $\mathcal{B}_{\mathrm{r}}(t)$. We then can recompose them to get the parameters for the quadrupedal locomotion. For validation purposes, we simulated a ambling step of the quadrupedal dynamics using the composed bipedal gaits. As shown in Fig. 5] we have the constraint wrench (ground reaction force) on toe 1 $\lambda_{1, z}$ and toe2 $\lambda_{2, z}$ of the quadruped matched with those corresponding external force to the bipedal dynamics. And the position terms matched as well. The details of controlling

TABLE I

COMPUTATING PERFORMANCE

\begin{tabular}{|l|l|l|l|l|l|}
\hline & gait1 & gait2 & gait3 & gait4 & amble \\
\hline frequency $(\mathrm{Hz})$ & 2.5 & 2.3 & 2.2 & 2.6 & 2.83 \\
\hline clearance $(\mathrm{cm})$ & 11 & 12 & 15 & 13 & 13 \\
\hline \# of iterations & 96 & 122 & 98 & 46 & 147 \\
\hline time of IPOPT (s) & 1.60 & 2.10 & 1.62 & 0.81 & 2.59 \\
\hline time of evaluation (s) & 1.94 & 3.24 & 2.10 & 0.94 & 2.86 \\
\hline NLP time(s) & 3.54 & 5.34 & 3.72 & 1.75 & 5.45 \\
\hline
\end{tabular}




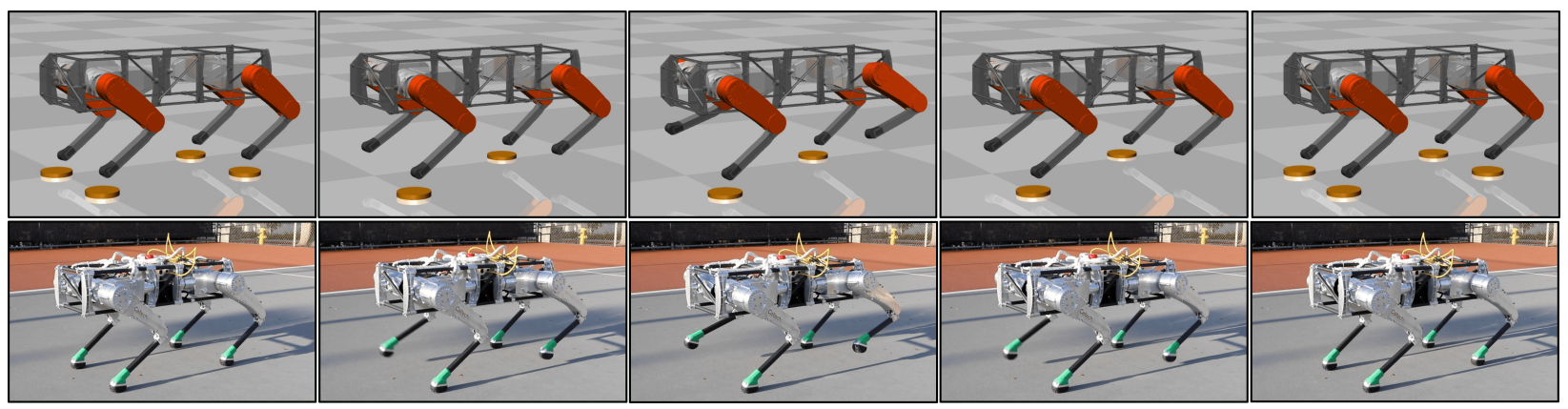

Fig. 6. Tiles of the experimental realization of the stepping in place gait4 on the Vision 60 quadruped.
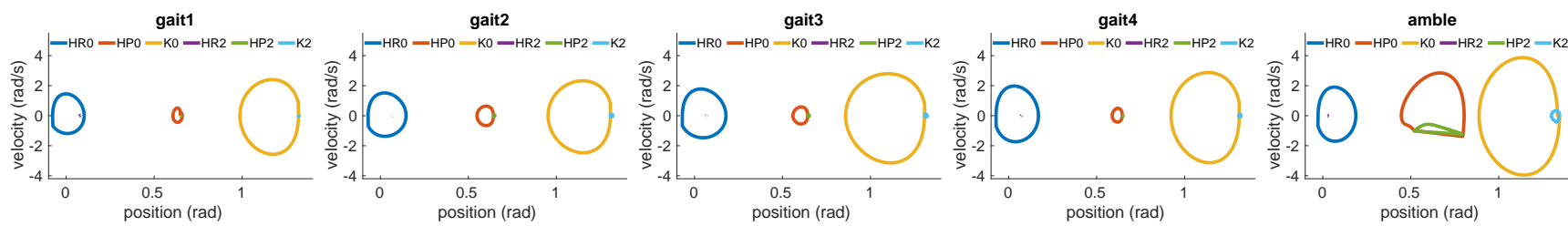

Fig. 7. Phase portrait of the four stepping gaits and a walking gait for the front bipedal robot subject to the connection force, when leg0 is the stance leg.

quadrupedal dynamics using a nonlinear controller can be found in [22].

In order to solve the optimization problem 25] efficiently, we used a toolbox FROST [19], [18], which parses a hybrid system control problem into a nonlinear programming (NLP) based on direct collocation techniques. It is worthwhile to mention that a key factor of the high efficiency of FROST comes from the implicit formulation of the dynamics. Matrix inversion is avoided in every step in this formulation due to its computational complexity: $\mathcal{O}\left(n^{3}\right)$, with $n$ the dimension of a matrix. Inspired by this, we remark the dynamics decomposition method proposed in this manuscript also only used differential algebra equations (DAEs) instead of ordinary differential equations (ODEs), which requires matrix inversion both for the inertia matrix and the closedloop controller formulation.

\section{A. Rapid gait generation and computing performance}

We now take advantage of the high efficiency of the decomposition based optimization to generate several different walking patterns for the front biped and then recompose them to obtain quadrupedal stepping-in-place behaviors. This is done by modifying constraint bounds in the gait generation NLP (25). For example, by changing the upper and lower bounds of time duration $T_{\min } \leq T \leq T_{\max }$, we can synthesize a higher or lower stepping frequency gait. Or, by changing the bounds of the nonstance foot height $\delta_{\min } \leq$ $h_{\mathrm{nsf}, z}\left(q_{\mathrm{f}}\right) \leq \delta_{\max }$, we can obtain a higher or lower stepping gait. In addition to the stepping behaviors, we also generated a bipedal walking gait that can be recomposed to a diagonally ambling gait for the quadruped with a speed of $0.35 \mathrm{~m} / \mathrm{s}$. This is done simply by releasing the constraint that the nonstance foot has to land on the same position that it lifts from. See Fig. 7 for the phase portrait of these optimal gaits and Figs. 9 and 6 for the experimental implementation.

The end result of the methods presented is the ability to rapidly generate quadrupedal gaits. This can be seen by considering the computing performance for each of the quadrupedal locomotion patterns generated, as is shown at Table I] To summarize, with the objective tolerance configured as $10^{-8}$, we have the average computation time as 3.96 second, and time per iteration averages 0.039 second. The computation test was conducted on a Linux laptop with an i7-6820HQ CPU @ $2.70 \mathrm{GHz}$ and $16 \mathrm{~GB}$ RAM. In a comparison with the regular full model based optimization methods from [22], the decomposition based optimization is an order of magnitude faster.

\section{IMPLEMENTATION}

One of the motivations for realizing rapid gaits using the full-order dynamics of the quadruped, i.e., without model simplifications, is to allow for the seamless translation of gaits from theory and simulation to hardware. With this as context, we first validated the gaits produced by the decomposition based optimization problem in simulation. These gaits includes four stepping in place and a diagonally symmetric ambling behavior. Then we conducted experiments with the same gaits and control infrastructure as in simulation in an outdoor environment (specifically, a tennis court). For both simulation and experiments, the implemented controller is a PD approximation of the inputoutput linearizing controllers used to track the time-based trajectories given by the optimization:

$$
u\left(q_{a}, \dot{q}_{a}, t\right)=-k_{1}\left(\dot{y}_{a}-\dot{\mathcal{B}}(t)\right)-k_{2}\left(y_{a}-\mathcal{B}(t)\right)
$$

for both the simulation and experiments. Note that the switching detection and the event functions are also given by the optimized trajectories, meaning the walking controller will switch to next step when $t=T$.

\section{A. Simulation}

In this paper, we used a third party physics engine, MuJoCo [30], to validate the five gaits and controllers pro- 


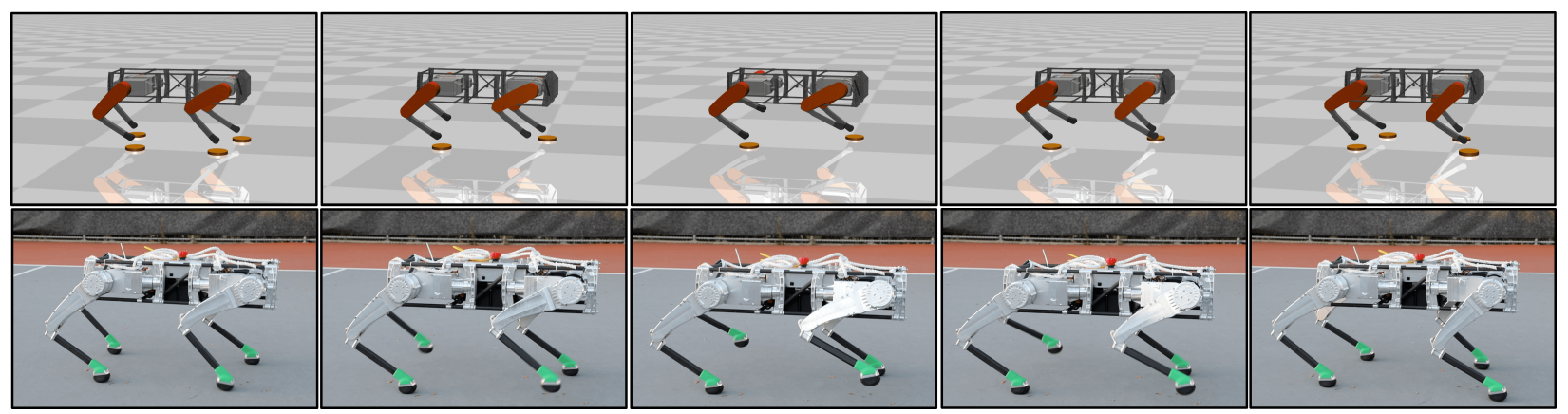

Fig. 8. Snapshots showing a full step of the diagonally symmetric ambling gait. The upper is from MuJoCo simulation, and the lower is from the Vision 60 quadruped ambling in a outdoor tennis court.

TABLE II

AVERAGE TORQUe INPUTS IN MUJOCO SIMULATION

\begin{tabular}{|l|l|l|l|l|l|}
\hline & gait1 & gait2 & gait3 & gait4 & amble \\
\hline $\bar{u}_{\mathrm{HR}}(\mathrm{N} \cdot \mathrm{m})$ & 7.80 & 9.23 & 10.27 & 8.68 & 8.06 \\
\hline $\bar{u}_{\mathrm{HP}}(\mathrm{N} \cdot \mathrm{m})$ & 6.78 & 9.14 & 10.71 & 6.64 & 7.27 \\
\hline $\bar{u}_{\mathrm{K}}(\mathrm{N} \cdot \mathrm{m})$ & 18.49 & 18.38 & 18.45 & 18.61 & 19.03 \\
\hline
\end{tabular}

duced by the dynamics decomposition based optimization. The averaged absolute joint torque inputs are reported in Table $[\mathrm{II}$, all of which are well within the hardware limitations. PD gains are chosen as $k_{p}=70,60,40$ and $k_{d}=$ $0.07,0.1,0.07$ for the hip roll, hip pitch, knee joints respectively. The ground coefficients are set as $0.8,0.05,0.0001$ for the sliding, torsional and rolling friction.

\section{B. Experiments}

Now that the quadrupedal dynamics have been decomposed into bipeds, optimized, recomposed and validated through MATLAB and MuJoCo simulations, we are ready to apply this method to the physical robot: the Vision 60 in Fig. 2 For all of the five optimal gaits including stepping and ambling, we set the PD gains to: $K_{p}=230,230,300$ and $k_{d}=5$ for the hip roll, hip pitch, knee joints, respectively. The result is that the Vision 60 quadruped can step and amble in a outdoor tennis court in a sustained fashion. Importantly, this is without any add-on layers of implementation or modification, i.e., without heuristics, and achieved by only changing the gait parameters $\alpha$ for each experiment (obtained from the different NLP optimization problems with different constraints and thus yielding different walking gaits). See [1] for the video of Vision 60 in both simulation and experimen $2^{2}$ and Fig. 8 for a side to side comparison of the simulated amble and experimental snapshots. We also recorded the averaged torque inputs in Table III for theses experiments. The tracking performance for the ambling gait in simulation and experiment are shown in Fig. 9 .

Finally, it is interesting to note that time-based control law (26) normally does not provide robustness against terrain dynamics, perturbations or uncertainty in the dynamics, due

\footnotetext{
2 In these five experiments, there is a consistent drifting-to-the-left phenomenon due to ordinary manufacturer defects and asymmetries in the robot. This could be mitigated by heuristically offsetting some joints. However, the goal of this paper was to show the direct implementation of those controllers without heuristics, thus no corrective action was taken.
}

TABLE III

AVERAGE TORQUE INPUTS IN EXPERIMENTS

\begin{tabular}{|l|l|l|l|l|l|}
\hline & gait1 & gait2 & gait3 & gait4 & amble \\
\hline $\bar{u}_{\mathrm{HR}}(\mathrm{N} \cdot \mathrm{m})$ & 5.04, & 4.83 & 4.16 & 5.14 & 7.11 \\
\hline $\bar{u}_{\mathrm{HP}}(\mathrm{N} \cdot \mathrm{m})$ & 3.65 & 5.24 & 5.26 & 3.77 & 6.28 \\
\hline $\bar{u}_{\mathrm{K}}(\mathrm{N} \cdot \mathrm{m})$ & 16.45 & 16.50 & 16.86 & 16.95 & 18.36 \\
\hline
\end{tabular}

to its open-loop nature. However, the fact that all of the trajectory based controllers remain stable in both simulation and experiment with an unified control setup speaks to the benefits of generating gaits using the full-body dynamics of the quadruped: even with an open-loop controller that does not leverage heuristics, the quadruped is still stable. Due to the decomposition of the full-body dynamics into bipeds, even through the quadruped is high-dimensional with complex contacts, we can generate gaits rapidly on the order of seconds.

\section{CONCLUSION}

In this paper, we decomposed the full body dynamics of a quadrupedal robot - the Vision 60 with $18 \mathrm{DOF}$ and 12 inputs - into two lower-dimensional bipedal systems that are subject to external forces. We are then able to solve the constrained dynamics of these bipeds quickly through the HZD optimization method, FROST, wherein the gaits can be recomposed to achieve locomotion on the original quadruped. The end result is the ability to rapidly generate walking gaits. Specifically, by changing a constraint, we are able to produce different bipedal and thus quadrupedal walking behaviors from stepping to ambling in 3.9 seconds on average. Furthermore, the implementation in simulation and experiments where successful using a single simple controller and without the need for additional heuristics.

Without sacrificing the model fidelity of the full-body dynamics of the quadruped, the ability to exactly decompose these dynamics into equivalent bipedal robots makes it possible to rapidly generate gaits that leverage the fullorder dynamics of the quadruped. Importantly, this allows for the rapid iteration of different gaits necessary for bringing quadrupeds into real-world environments. Moreover, the fact that these gaits can be generated on the order of seconds suggests that with code optimization on-board and real-time gait generation may be possible in the near future. The goal is 

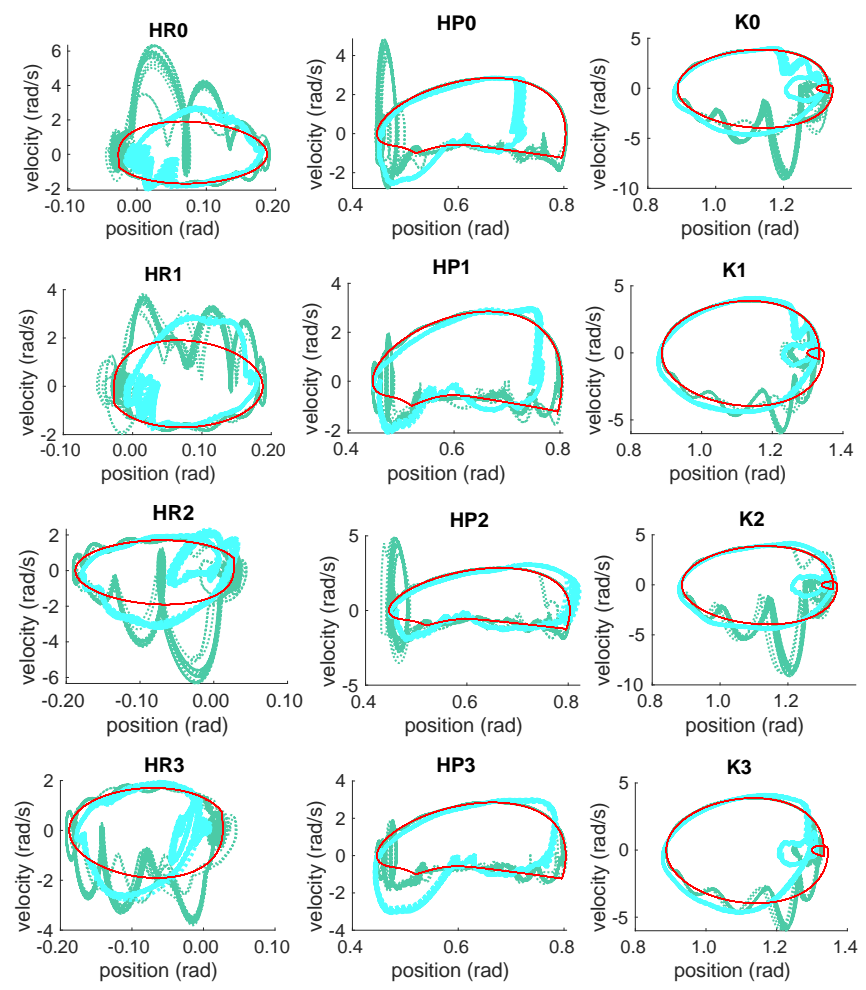

Fig. 9. Tracking performance of the optimal ambling gait (in red) vs. the MuJoCo simulated result (in green) vs. the experimental data (in cyan) in the form of phase portrait using 18 seconds' data. HR is short for the hip roll joint, $\mathrm{HP}$ is for the hip pitch joint and $\mathrm{K}$ is for the knee joint.

to ultimately use this method to realize a variety of different dynamic locomotion behaviors on quadrupeds.

\section{REFERENCES}

[1] Experimental video of Vision 60 using the composed gaits, https: //youtu.be/nBRZFqiHaBY

[2] K. Akbari Hamed, B. Buss, and J. Grizzle. Exponentially stabilizing continuous-time controllers for periodic orbits of hybrid systems: Application to bipedal locomotion with ground height variations. The International Journal of Robotics Research, 35(8):977-999, 2016.

[3] K. Akbari Hamed, W.-L. Ma, and A. D. Ames. Dynamically stable $3 \mathrm{~d}$ quadrupedal walking with multi-domain hybrid system models and virtual constraint controllers. In 2019 American Control Conference (ACC), pages 4588-4595, July 2019.

[4] A. Ames. Human-inspired control of bipedal walking robots. Automatic Control, IEEE Transactions on, 59(5):1115-1130, May 2014.

[5] A. Ames, K. Galloway, K. Sreenath, and J. Grizzle. Rapidly exponentially stabilizing control Lyapunov functions and hybrid zero dynamics. Automatic Control, IEEE Transactions on, April 2014.

[6] G. Bledt, M. J. Powell, B. Katz, J. Di Carlo, P. M. Wensing, and S. Kim. Mit cheetah 3: Design and control of a robust, dynamic quadruped robot. In 2018 IEEE/RSJ International Conference on Intelligent Robots and Systems (IROS), pages 2245-2252, Oct 2018.

[7] C. Boussema, M. J. Powell, G. Bledt, A. J. Ijspeert, P. M. Wensing, and S. Kim. Online gait transitions and disturbance recovery for legged robots via the feasible impulse set. IEEE Robotics and Automation Letters, 4(2):1611-1618, April 2019.

[8] C. Boussema, M. J. Powell, G. Bledt, A. J. Ijspeert, P. M. Wensing, and S. Kim. Online gait transitions and disturbance recovery for legged robots via the feasible impulse set. IEEE Robotics and Automation Letters, 4(2):1611-1618, April 2019.

[9] C. Dario Bellicoso, F. Jenelten, P. Fankhauser, C. Gehring, J. Hwangbo, and M. Hutter. Dynamic locomotion and whole-body control for quadrupedal robots. In 2017 IEEE/RSJ International Conference on Intelligent Robots and Systems (IROS), pages 33593365, Sep. 2017.
[10] A. De. Modular hopping and running via parallel composition. 2017.

[11] A. De and D. E. Koditschek. Parallel composition of templates for tail-energized planar hopping. In 2015 IEEE International Conference on Robotics and Automation (ICRA), pages 4562-4569, May 2015.

[12] A. De and D. E. Koditschek. Vertical hopper compositions for preflexive and feedback-stabilized quadrupedal bounding, pacing, pronking, and trotting. The International Journal of Robotics Research, 37(7):743-778, 2018.

[13] R. Featherstone. Rigid Body Dynamics Algorithms. Kluwer international series in engineering and computer science: Robotics. Springer, 2008.

[14] J. W. Grizzle, G. Abba, and F. Plestan. Asymptotically Stable Walking for Biped Robots: Analysis via Systems with Impulse Effects. IEEE Trans. on Automatic Control, 46(1):51-64, Jan. 2001.

[15] J. W. Grizzle, C. Chevallereau, R. W. Sinnet, and A. D. Ames. Models, feedback control, and open problems of 3D bipedal robotic walking. Automatica, 50(8):1955 - 1988, 2014.

[16] A. Hereid, E. Cousineau, C. Hubicki, and A. D. Ames. 3D dynamic walking with underactuated humanoid robots: A direct collocation framework for optimizing hybrid zero dynamics. In IEEE International Conference on Robotics and Automation, 2016.

[17] A. Hereid, E. A. Cousineau, C. M. Hubicki, and A. D. Ames. 3d dynamic walking with underactuated humanoid robots: A direct collocation framework for optimizing hybrid zero dynamics. In IEEE International Conference on Robotics and Automation (ICRA). IEEE, 2016.

[18] A. Hereid, O. Harib, R. Hartley, Y. Gong, and J. W. Grizzle. Rapid bipedal gait design using C-FROST with illustration on a cassie-series robot. CoRR, abs/1807.06614, 2018.

[19] A. Hereid, C. M. Hubicki, E. A. Cousineau, and A. D. Ames. Dynamic humanoid locomotion: A scalable formulation for HZD gait optimization. IEEE Transactions on Robotics, 2018.

[20] A. Hereid, S. Kolathaya, and A. D. Ames. Online optimal gait generation for bipedal walking robots using legendre pseudospectral optimization. In 2016 IEEE 55th Conference on Decision and Control (CDC), pages 6173-6179, Dec 2016.

[21] S. Kajita, K. Tani, and A. Kobayashi. Dynamic walk control of a biped robot along the potential energy conserving orbit. In IEEE International Workshop on Intelligent Robots and Systems, Towards a New Frontier of Applications, pages 789-794 vol.2, July 1990.

[22] W.-L. Ma, K. Akbari Hamed, and A. D. Ames. First steps towards full model based motion planning and control of quadrupeds: A hybrid zero dynamics approach. In 2019 IEEE International Conference on Intelligent Robots and Systems (IROS), Macau, China, 2019.

[23] W.-L. Ma, S. Kolathaya, E. R. Ambrose, C. M. Hubicki, and A. D. Ames. Bipedal robotic running with durus-2d: Bridging the gap between theory and experiment. In Proceedings of the 20th International Conference on Hybrid Systems: Computation and Control, HSCC '17, pages 265-274, New York, NY, USA, 2017. ACM.

[24] B. Morris and J. W. Grizzle. Hybrid invariant manifolds in systems with impulse effects with application to periodic locomotion in bipedal robots. IEEE Transactions on Automatic Control, 54(8):1751-1764.

[25] K. N. Murphy and M. H. Raibert. Trotting and Bounding in a Planar Two-legged Model, pages 411-420. Springer US, Boston, MA, 1985.

[26] M. H. Raibert, B. H. Brown, M. Chepponis, J. Koechling, J. Hodgins, D. Dustman, K. W. rennan, D. Barrett, C. Thompson, J. Hebert, W. Lee, and L. Borvansky. Dynamically stable legged locomotion (september 1985-septembers1989). pages 49-77.

[27] J. Reher, W.-L. Ma, and A. D. Ames. Dynamic walking with compliance on a cassie bipedal robot. 2019 18th European Control Conference (ECC), pages 2589-2595, 2019.

[28] S. Sastry. Nonlinear systems: analysis, stability, and control, volume 10. Springer New York, 1999.

[29] K. Sreenath, H.-W. Park, I. Poulakakis, and J. W. Grizzle. Compliant hybrid zero dynamics controller for achieving stable, efficient and fast bipedal walking on MABEL. The International Journal of Robotics Research, 30(9):1170-1193, Aug. 2011.

[30] E. Todorov. Convex and analytically-invertible dynamics with contacts and constraints: Theory and implementation in mujoco. In 2014 IEEE International Conference on Robotics and Automation (ICRA), pages 6054-6061, May 2014.

[31] E. R. Westervelt, J. W. Grizzle, C. Chevallereau, J. H. Choi, and B. Morris. Feedback Control of Dynamic Bipedal Robot Locomotion. Control and Automation. CRC Press, Boca Raton, June 2007. 